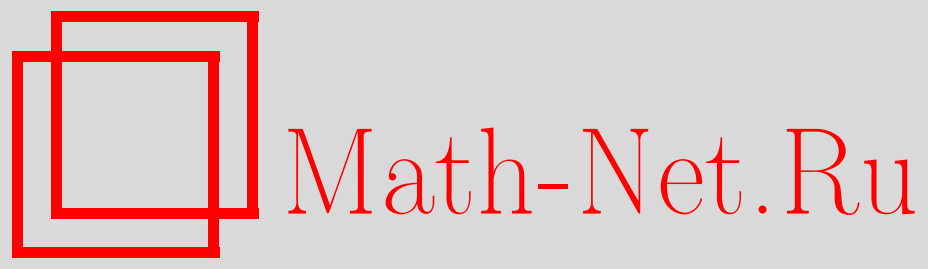

В. М. Бухштабер, И. В. Нетай, Функциональное уравнение Хирцебруха и эллиптические функции уровня $d$, Функи. анализ и его прил., 2015, том 49, выпуск 4, 1-17

DOI: https://doi.org/10.4213/faa3219

Использование Общероссийского математического портала Math$\mathrm{Net.Ru} \mathrm{подразумевает,} \mathrm{что} \mathrm{вы} \mathrm{прочитали} \mathrm{и} \mathrm{согласны} \mathrm{с} \mathrm{пользователь-}$ ским соглашением

http://www . mathnet.ru/rus/agreement

Параметры загрузки:

IP : 54.147 .182 .235

26 апреля 2023 г., 15:04:23

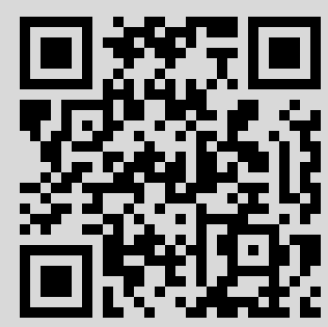


Функционалъный анализ и его приложения

2015, т. 49, вып. 4, с. 1-17

УДК $517.9+515.178 .13+515.14$

\title{
Функциональное уравнение Хирцебруха и эллиптические функции уровня $d$
}

\author{
(C) 2015. В. М. БУХштАБеР, И. В. НетАй*
}

\section{Посвящается замечательному математику Александру Петровичу Веселову в связи с его 60-летием}

\begin{abstract}
Функция $f(x)$ комплексного переменного $x$, регулярная в окрестности точки $x=0$ и такая, что $f(0)=0, f^{\prime}(0)=1$, называется $n$-жесткой, если сумма вычетов функции $\prod_{i=0}^{n} 1 / f\left(x-x_{i}\right)$ не зависит от выбора не совпадающих точек $x_{0}, \ldots, x_{n}$ в малой окрестности точки $x=0$. Ряд, задающий $n$-жесткую функцию, определяется функциональным уравнением. Это уравнение мы называем $n$-уравнением Хирцебруха. Каждая эллиптическая функции уровня $d$, где $d-$ делитель числа $n+1$, является $n$-жесткой. Описание многообразия всех 2-жестких функций получено совсем недавно. Основным результатом настоящей работы является описание многообразия всех 3-жестких функций.
\end{abstract}

\section{Введение}

Настоящая работа тематически и в направлении приложений связана с работами [1]-[3].

Рассмотрим функцию $f(x)$ комплексного переменного $x$, регулярную в окрестности точки $x=0$ и такую, что $f(0)=0$ и $f^{\prime}(0)=1$. Положим

$$
F(x)=F\left(x ; x_{0}, \ldots, x_{n}\right)=\prod_{i=0}^{n} \frac{1}{f\left(x-x_{i}\right)} .
$$

Определение 0.1. Пусть $U$ - окрестность точки $x$, в которой функция $f(x)$ не имеет нулей, кроме $x=0$. Функция $f(x)$ называется $n$-жесткой, если сумма вычетов функции $F(x)$ в $U$ не зависит от выбора не совпадающих точек $x_{0}, \ldots, x_{n} \in U$. Обозначим эту сумму вычетов через $C_{n}$.

Можно показать, что функция $f(x)$ является $n$-жесткой тогда и только тогда, когда ряд $x+\sum_{k \geqslant 1} f_{k} x^{k+1}$, задающий функцию $f(x)$ в малой окрестности точки $x=0$, удовлетворяет функциональному уравнению

$$
\sum_{j=0}^{n} \prod_{i \neq j} \frac{1}{f\left(x_{j}-x_{i}\right)}=C_{n}
$$

где $C_{n}$ зависит от $f$, но не зависит от выбора точек $x_{0}, \ldots, x_{n}$.

Уравнение (1) играет важную роль в теории родов Хирцебруха и в их приложениях (см. [8], [14] и §4). Это дало нам основание назвать его $n$-уравнением

* Исследование выполнено при финансовой поддержке Российского научного фонда (проект 14-50-00005) в Математическом институте им. В. А. Стеклова РАН. 
Хирцебруха. В $\S 4$ мы вводим определение $n$-жестких родов Хирцебруха и описываем их связь с решениями $n$-уравнений Хирцебруха.

Хорошо известно, что функция

$$
f(x)=\frac{e^{a x}-e^{b x}}{a e^{b x}-b e^{a x}}
$$

является $n$-жесткой для всех $n$ и всех значений параметров $a$ и $b$ (см. [8]). Более того, недавно было показано, что функция $f(x)$ является $n$-жесткой для всех $n$ тогда и только тогда, когда она имеет вид (2) (см. [5]).

Разложение в ряд функции (2) задает знаменитый 2-параметрический род Тодда (см. [4], [8], [14] и §4). Эта функция естественно возникает в различных областях математики. Например, имеет место разложение

$$
\frac{e^{a x}-e^{b x}}{a e^{b x}-b e^{a x}}=\sum_{k=0}^{\infty} \sum_{i+j=k+1} A(i, j) a^{i} b^{j} \frac{x^{k+1}}{(k+1) !},
$$

где $A(i, j)$ - число перестановок множества из $k+1$ элементов, имеющих $i$ возрастаний и $j$ убываний (см. [15]). В перечислительной комбинаторике и торической топологии эта функция возникает благодаря разложению

$$
\frac{e^{a x}-e^{b x}}{a e^{b x}-b e^{a x}}=\sum_{k \geqslant 0} H\left(\mathrm{Pe}^{k}\right) \frac{x^{k+1}}{(k+1) !},
$$

где $H\left(\mathrm{Pe}^{k}\right)$ есть $H$-полином $k$-мерного пермутоэдра (см. [14]). Для данного $n$ функция (2) удовлетворяет $n$-уравнению Хирцебруха с константой

$$
C_{n}=(-1)^{n} \frac{a^{n+1}-b^{n+1}}{a-b},
$$

где $C_{n}=C_{n}(f)$. Для ряда $f^{-1}(x)$, функционально обратного к ряду, задающему функцию $(2)$, имеет место формула

$$
f^{-1}(x)=x+\sum_{n \geqslant 1} C_{n} \frac{x^{n+1}}{n+1},
$$

где $C_{n}=C_{n}(f)$. При алгебротопологической интерпретации решений функционального уравнения Хирцебруха (см. §4) формула (3) переходит в формулу С. П. Новикова (см. $\left.\left[16, \S 4^{\prime}\right]\right)$.

Классические методы теории эллиптических функций позволяют непосредственно показать (см. [7], [8] и приложение А), что эллиптические функции уровня $d$ являются $n$-жесткими, если $d-$ делитель числа $n+1$.

Совсем недавно был получен следующий результат (см. [13]).

Теорема 0.2. Пусть $f(x)$ - некоторая 2-энесткая функиия. Тогда при $C_{2} \neq 0$ она имеет вид (2), а при $C_{2}=0$ является эллиптической функиией уровня 3 .

Следствие 0.3. Эквивалентны следующие условия:

(i) функиия $f(x)$ является 2-жесткой;

(ii) функиия $f(x)$ является $(3 n-1)$-жесткой для любого $n$.

Основной результат настоящей работы: 
Теорема 0.4. Пусть $f(x)$ - некоторая 3-жесткая функиия. Тогда при $C_{3} \neq 0$ она имеет вид (2), а при $C_{3}=0$ представляет собой эллиптический синус Якоби $\operatorname{sn}(x)$ или эллиптическую функцию уровня 4.

Следствие 0.5. Эквивалентны следующие условия:

(i) функиия $f(x)$ является 3 -жесткой;

(ii) функиия $f(x)$ является $(4 n-1)$-жесткой для любого $n$.

Доказательства теорем 0.2 и 0.4 оказались нетривиальными и существенно используют результаты теории симметрических полиномов, в том числе аппарат полиномов Шура. Мы развиваем общий метод, который сначала демонстрируем на доказательстве теоремы 0.2 (см. теорему 2.1), а затем этим методом проводим детальное доказательство теоремы 0.4 (см. теорему 3.2 ).

В приложениях А и В собраны необходимые определения и результаты об эллиптических функциях уровня $d$.

\section{§1. Параметризация решений уравнения Хирцебруха}

Положим

$$
h(x)=\frac{1}{f(x)}=\frac{1}{x}+\sum_{k=0}^{\infty} h_{k+1} x^{k} .
$$

Можно рассматривать $h_{k+1}$ как координатные функции на пространстве решений уравнения (1), сопоставляющие формальному ряду $h(x)$ над коммутативным ассоциативным кольцом $R$ коэффициент при $x^{k}$. Обозначим через $\mathscr{S}_{n, f}$ левую часть уравнения (1),

$$
\mathscr{S}_{n, f}=\sum_{k=0}^{n} \prod_{i \neq k} \frac{1}{f\left(x_{k}-x_{i}\right)} .
$$

Ниже (см. лемму 1.4) мы покажем, что для любого формального ряда $f(x)$ с $f(0)=0, f^{\prime}(0)=1$ это выражение задается формальным рядом.

Поскольку выражение $\mathscr{S}_{n, f}$ симметрично относительно перестановок переменных $x_{0}, \ldots, x_{n}$, этот формальный ряд можно разложить, используя аддитивный базис полиномов Шура в кольце симметрических полиномов:

$$
\mathscr{S}_{n, f}=\sum_{\lambda} p_{n, \lambda} s_{\lambda}
$$

где $s_{\lambda}-$ полином Шура (см. определение 1.6), соответствующий диаграмме Юнга $\lambda$, а $p_{n, \lambda}-$ некоторый полином от переменных $h_{i}$. Правая часть уравнения (1) имеет вид $C_{n} \cdot s_{\varnothing}$. Таким образом, мы получаем следующий результат.

Теорема 1.1. Функииональное уравнение (1) равносильно системе полиномиальных уравнений $\left\{p_{n, \lambda}=0\right\}$ для всех диаграмм Юнга $\lambda \neq \varnothing$ на коэфбиииенты $h_{i} \in R$. При этом $C_{n}=p_{n, \varnothing}$.

Следствие 1.2. Пространство решений уравнения (1) можно отождествить со схемой

$$
\mathscr{M}_{n}:=\operatorname{Spec}\left(R\left[h_{1}, h_{2}, \ldots\right] / \operatorname{Rad}(I)\right),
$$

где $\operatorname{Rad}(I)-$ радикал идеала $I=\left(p_{n, \lambda}\right)_{\lambda \neq \varnothing}$.

Будем говорить, что решение $h(x)=1 / f(x)$ задает точку схемы $\mathscr{M}_{n}$. 
Пример 1.3. Рассмотрим случай $n=1$. Легко видеть, что идеал $\left(p_{1, \lambda}\right)_{\lambda \neq \varnothing}$ порожден всеми $h_{2 k+1}$ для $k \geqslant 1$, если $R$ не имеет 2 -кручения. Таким образом,

$$
\mathscr{M}_{1}=\mathbb{A}_{R}^{\infty}=\operatorname{Spec} R\left[h_{1}, h_{2}, h_{4}, h_{6}, \ldots\right]
$$

является бесконечномерным аффинным пространством над кольцом $R$.

Ниже мы увидим, что если $n \neq 1$ и $R$ - поле нулевой характеристики, то $\mathscr{M}_{n}(R)$ является многообразием. В работе [13] показано, что $\mathscr{M}_{2}(\mathbb{Q})$ является объединением двух аффинных плоскостей, пересекающихся по конике. Ниже мы опишем структуру многообразия $\mathscr{M}_{3}$ над полем нулевой характеристики, параметризующего 3-жесткие функции, а также опишем эти функции.

Введем дифференциальные разностные операторы $\partial_{i}=\left(x_{i}-x_{i-1}\right)^{-1}\left(1-\tau_{i}\right)$, где $\tau_{i}, i=1, \ldots, n$, действует на кольце полиномов $R\left[x_{0}, \ldots, x_{n}\right]$ как кольцевой автоморфизм, переставляющий переменные $x_{i-1}$ и $x_{i}$. Хорошо известно, что $\partial_{i} P$ является полиномом для любого полинома $P$. При этом $\partial_{i} P$ инвариантен относительно перестановки $\tau_{i}$ и $\partial_{i} P=0$, если и только если $P$ инвариантен относительно этой перестановки. Легко проверить, что

$$
\partial(P \cdot Q)=\partial_{i}(P) \cdot Q+\tau_{i}(P) \cdot \partial_{i}(Q) .
$$

Если $\tau_{i}(Q)=Q$, то $\partial_{i}(P \cdot Q)=\partial_{i}(P) \cdot Q$. Следовательно, операторы $\partial_{i}$ являются $\tau_{i}$-дифференциальными операторами на кольце полиномов как на модуле над кольцом симметрических полиномов.

Обозначим через $\mathfrak{S}_{n+1}$ симметрическую группу перестановок переменных $x_{0}, \ldots, x_{n}$, а через $\operatorname{Sym}_{n+1}=R\left[x_{0}, \ldots, x_{n}\right]^{\mathfrak{S}_{n+1}}$ кольцо симметрических полиномов. Нам потребуется оператор

$$
L=\frac{1}{\Delta} \sum_{\sigma \in \mathfrak{S}_{n+1}}(-1)^{\sigma} \sigma,
$$

где $\Delta=\prod_{i<j}\left(x_{i}-x_{j}\right)$. Хорошо известно (см. [6]), что

$$
L=\left(\partial_{1} \cdots \partial_{n}\right)\left(\partial_{1} \cdots \partial_{n-1}\right) \cdots\left(\partial_{1} \partial_{2}\right) \partial_{1} .
$$

Нетрудно проверить, что $L(1)=0$ и $L(P \cdot Q)=L(P) \cdot Q$, если полином $Q$ симметрический. Имеет место важное соотношение $L \Delta=(n+1)$ !, поэтому $L(\Delta Q)=(n+1) ! Q$ для любого симметрического полинома $Q$. Следовательно, оператор $\frac{1}{(n+1) !} L \Delta$ является проектором $R\left[x_{0}, \ldots, x_{n}\right] \rightarrow \operatorname{Sym}_{n+1}$.

Действие оператора $L$ продолжается на поле рациональных функций от $x_{0}, \ldots, x_{n}$. Легко проверить, что это продолжение обладает всеми перечисленными свойствами, в частности, $L(P \cdot Q)=L(P) \cdot Q$ для любой симметрической рациональной функции $Q$ и любой рациональной функции $P$.

Лемма 1.4. Для любого формального ряда $f(x)=x+O\left(x^{2}\right)$ выражение $\mathscr{S}_{n, f}(x)$ задает формальный ряд.

Доказательство. Положим $\tilde{h}(x):=x \cdot h(x)$. Очевидно, что выражение $\mathscr{S}_{n, f}$ задает симметрическую рациональную функцию; следовательно, $L\left(\Delta \mathscr{S}_{n, f}\right)=$ 
$(n+1) ! \mathscr{S}_{n, f} \cdot$ Таким образом,

$$
\begin{aligned}
\mathscr{S}_{n, f} & =\frac{1}{(n+1) !} L\left(\Delta \mathscr{S}_{n, f}\right)=\frac{1}{(n+1) !} L\left(\Delta \cdot \sum_{k=0}^{n} \prod_{i \neq k} \frac{1}{f\left(x_{k}-x_{i}\right)}\right) \\
& =\frac{1}{(n+1) !} L\left(\sum_{i=0}^{n} \varepsilon_{i} \prod_{i \neq k} \tilde{h}\left(x_{i}-x_{k}\right) \prod_{\substack{i<j \\
i, j \neq k}}\left(x_{i}-x_{j}\right)\right) \in R\left[\left[x_{0}, \ldots, x_{n}\right]\right],
\end{aligned}
$$

где $\varepsilon_{i}= \pm 1$.

Согласно лемме 1.4, определена константа $C_{n}=\mathscr{S}_{n, f}(0)$.

Определение 1.5. Формальный ряд $f(x)$ называется специальным для $n$-уравнения Хирцебруха, если $C_{n}=0$.

Пусть $\lambda=\left\{\lambda_{1} \geqslant \cdots \geqslant \lambda_{n}\right\}$ - диаграмма Юнга. Положим

$$
\Delta_{\lambda}=\left|\begin{array}{ccc}
x_{1}^{\lambda_{1}} & \ldots & x_{n}^{\lambda_{1}} \\
\vdots & \ddots & \vdots \\
x_{1}^{\lambda_{n}} & \ldots & x_{n}^{\lambda_{n}}
\end{array}\right| .
$$

Нетрудно проверить, что полином $\Delta_{\lambda+\delta}$ делится на полином $\Delta_{\delta}$, где $\delta=(n-1$, $\ldots, 1,0)$.

Определение 1.6. Полином Шура, соответствующий диаграмме $\lambda$, задается формулой

$$
s_{\lambda}=\Delta_{\lambda+\delta} / \Delta_{\delta}
$$

Полином $s_{\lambda}$ как отношение двух кососимметрических полиномов является симметрическим полиномом. Известно (см., например, [6]), что множество полиномов Шура $s_{\lambda}\left(x_{1}, \ldots, x_{n}\right)$, таких, что $|\lambda|=\sum \lambda_{i}=m$ и $\operatorname{ht}(\lambda) \leqslant n$, где $\operatorname{ht}(\lambda)$ - число ненулевых строк в диаграмме Юнга $\lambda$, является аддитивным базисом пространства симметрических полиномов степени $m$ для любых $m$. Положим $x^{\lambda}:=x_{1}^{\lambda_{1}} \cdots x_{n}^{\lambda_{n}}$ для диаграммы Юнга $\lambda$. Тогда имеет место важная формула $L x^{\lambda+\delta}=s_{\lambda}$. Следовательно, если моном $x^{\lambda}$ симметричен относительно какойлибо перестановки $\tau_{i}$, то $L x^{\lambda}=0$.

В пространстве полиномов от $n$ переменных выберем мономиальный базис $\left\{x^{\omega}, \omega \in \mathbb{Z}_{\geqslant 0}^{n}\right\}$. В пространстве симметрических полиномов, являющемся образом оператора $L$, выберем базис $\left\{s_{\lambda}\right\}$ полиномов Шура. Тогда оператор $L$ задается матрицей

$$
L_{\omega, \lambda}= \begin{cases}(-1)^{\sigma}, & \sigma(\omega)=\lambda-\delta \\ 0 & \text { в противном случае. }\end{cases}
$$

Вернемся к разложению (4). Легко видеть, что полином $p_{n, \lambda}$ является однородным полиномом степени $|\lambda|+n$ относительно градуировки $\operatorname{deg} h_{k}=k$. Следовательно, он линеен по переменной $h_{|\lambda|-n}$ и зависит только от переменных $h_{i}$, где $i \leqslant n+|\lambda|$. Рассмотрим более подробно полином $p_{n, \lambda}$, где $\lambda=(m)$ состоит из одной строки.

Лемма 1.7. Для любъх $n$ и $m$ коэфбициент при $h_{m+n}$ у полинома $p_{n,(m)}$ равен $n(-1)^{n+m}+(-1)^{n}\left(\begin{array}{c}n+m-1 \\ m\end{array}\right)$. 
Доказательство. Возьмем некоторую подгруппу $\mathfrak{G} \subseteq \mathfrak{S}_{n+1}$ в группе перестановок переменных $x_{0}, \ldots, x_{n}$. Пусть полином $D$ кососимметричен относительно подгруппы $\mathfrak{G}$, т. е. $\sigma D=(-1)^{\sigma} D$ для любого $\sigma \in \mathfrak{G}$. Тогда для любого полинома $Q$ имеем равенство

$$
L(D Q)=\frac{1}{|\mathfrak{G}|} L\left(\sum_{\sigma \in \mathfrak{G}}(-1)^{\sigma} \sigma(D Q)\right)=\frac{1}{|\mathfrak{G}|} L\left(D \sum_{\sigma \in \mathfrak{G}} \sigma Q\right) .
$$

Пусть теперь $S-$ полином, симметричный относительно подгруппы $\mathfrak{G}$, т. е. $\sigma S=S$ для любого $\sigma \in \mathfrak{G}$. Тогда для любого полинома $Q$ имеет место равенство

$$
L(S Q)=\frac{1}{|\mathfrak{G}|} L\left(\sum_{\sigma \in \mathfrak{G}}(-1)^{\sigma} \sigma(S Q)\right)=\frac{1}{|\mathfrak{G}|} L\left(S \sum_{\sigma \in \mathfrak{G}}(-1)^{\sigma} \sigma Q\right) .
$$

Обозначим через $\vartheta$ циклическую перестановку набора $\left(x_{0}, \ldots, x_{n}\right)$. Использовав формулу (5) в случае $D=\Delta, \mathfrak{G}=\langle\vartheta\rangle$, получаем:

$$
L\left(\prod_{\substack{i<j \\ i, j \neq 0}}\left(x_{i}-x_{j}\right) \prod_{i=1}^{n} \tilde{h}\left(x_{i}-x_{0}\right)\right)=n ! C_{n} .
$$

Используем формулу (6), взяв $Q=x_{1}^{n-1} x_{2}^{n-2} \cdots x_{n-1}, S=\prod_{i=1}^{n} \tilde{h}\left(x_{i}-x_{0}\right)$. В качестве $\mathfrak{G}$ возьмем группу перестановок поднабора $\left(x_{1}, \ldots, x_{n}\right)$. Тогда из $(7)$ получим уравнение, равносильное уравнению (1):

$$
L\left(x_{1}^{n-1} x_{2}^{n-2} \cdots x_{n-1} \prod_{i=1}^{n} \tilde{h}\left(x_{i}-x_{0}\right)\right)=C_{n} .
$$

Разложим выражение, стоящее в скобках в левой части уравнения (8) по базису мономов, применим к нему оператор $L$ и получим разложение по базису полиномов Шура. Вклад в коэффициент при полиноме Шура $s_{(m)}$ дают мономы, имеющие набор степеней $(m+n, n-1, \ldots, 0)$. Поскольку в выражении

$$
\prod_{i=1}^{n} \tilde{h}\left(x_{i}-x_{0}\right)=\prod_{i=1}^{n} \sum_{k=0}^{\infty} h_{k}\left(x_{i}-x_{0}\right)^{k},
$$

где $h_{0}=1$, нас интересует только коэффициент при $h_{n+m}$, мы можем перейти к сумме $\sum_{i=1}^{n} h_{n+m}\left(x_{i}-x_{0}\right)^{m+n}$. Вклад в коэффициент при $h_{m+n}$ в этой сумме дают мономы с набором степеней $(m+n, n-1, \ldots, 1,0)$ в выражении

$$
x_{1}^{n-1} x_{2}^{n-2} \cdots x_{n-1} \sum_{i=1}^{n}\left(x_{i}-x_{0}\right)^{n+m} .
$$

Таким образом, мы хотим перебрать способы получить из набора степеней $(0$, $n-1, \ldots, 1,0)$ набор $(m+n, n-1, \ldots, 1,0)$, добавив к первой и какой-нибудь другой координате по неотрицательному целому числу, сумма которых равна $n+m$. Мы можем сделать это, добавив $n+m$ к первой координате или добавить $n-i$ к степени переменной $x_{0}$ и $m+i$ к степени переменной $x_{i}$ для каждого 
$i$ от 1 до $n$. Таким образом, мы получаем следующие мономы, каждый со своим коэффициентом:

$$
\begin{gathered}
n(-1)^{m+n} x_{0}^{m+n} x_{1}^{n-1} \cdots x_{n-1}, \quad(-1)^{n-1}\left(\begin{array}{c}
n+m \\
n-1
\end{array}\right) x_{1}^{n+m} x_{0}^{n-1} \cdots x_{n-1}, \\
(-1)^{n-2}\left(\begin{array}{c}
n+m \\
n-2
\end{array}\right) x_{2}^{m+n} x_{1}^{n-1} x_{0}^{n-2} \cdots x_{n-1}, \ldots,(-1)^{2}\left(\begin{array}{c}
n+m \\
2
\end{array}\right) x_{n-2}^{m+n} x_{1}^{n-1} \cdots x_{0}^{2} x_{n-1}, \\
(-1)\left(\begin{array}{c}
m+n \\
1
\end{array}\right) x_{n-1}^{m+n} x_{1}^{n-1} \cdots x_{0}, \quad x_{n}^{m+n} x_{1}^{n-1} \cdots x_{n-1} .
\end{gathered}
$$

Мы видим, что в первом мономе индексы переменных возрастают, а в остальных их порядок отличается от возрастающего одной транспозицией. Таким образом, коэффициент при $h_{m+n}$ в полиноме $p_{(m)}$ равен

$$
\begin{array}{r}
(-1)^{m+n} n+(-1)^{n}\left(\begin{array}{c}
n+m \\
n-1
\end{array}\right)+(-1)^{n-1}\left(\begin{array}{c}
n+m \\
n-2
\end{array}\right)+\cdots+\left(\begin{array}{c}
m+n \\
1
\end{array}\right)-\left(\begin{array}{c}
m+n \\
0
\end{array}\right) \\
=(-1)^{m+n} n+(-1)^{n}\left(\begin{array}{c}
n+m-1 \\
n-1
\end{array}\right)=(-1)^{n+m} n+(-1)^{n}\left(\begin{array}{c}
n+m-1 \\
m
\end{array}\right) .
\end{array}
$$

Таким образом, лемма доказана.

Лемма 1.8. Если кольцо коэфбициентов $R$ является полем нулевой характеристики, то коэфбициент при мономе $h_{m+n}$ в полиноме $p_{n,(m)}$ не равен нулю при $m>1$ и $n>1$.

Доказательство. Пусть $C_{m, n}-$ искомый коэффициент. Тогда $C_{m, n}=$ $(-1)^{m+n} n+(-1)^{n}\left(\begin{array}{c}m+n-1 \\ m\end{array}\right)$ согласно лемме 1.7. Заметим, что последовательность $\left\{(-1)^{n} C_{m, n}\right\}$ является строго возрастающей по $m$ отдельно при четных и нечетных натуральных $m$ и при $n>1$. Легко видеть, что $(-1)^{n} C_{m, n}>0$ при любом четном $m$. Легко также убедиться, что $C_{1, n}=0$, и поэтому $C_{m, n}>0$ и при нечетном $m$.

Далее будем считать, что кольцо $R$ является полем нулевой характеристики.

Теорема 1.9. При $n>1$ схема $\mathscr{M}_{n}(R)$ является многообразием.

Доказательство. Мы хотим убедиться, что это схема конечного типа. Как мы установили выше, она задается всеми полиномами $p_{n, \lambda}$ для всех диаграмм Юнга $\lambda \neq \varnothing$ в аффинном пространстве с координатами $h_{k}$ для $k \in \mathbb{N}$. Поскольку $p_{n,(m)}$ линейно зависит от $h_{m+n}$ при $m>1$, мы можем исключить переменные $h_{k}$ с $k>n+1$. Выражая таким образом в полиномах $p_{n, \lambda}$, где диаграмма $\lambda$ имеет более одной строки, переменные $h_{k}$ с $k>n+1$ через $h_{1}, \ldots, h_{n+1}$, мы получаем, что

$$
\mathscr{M}_{n}=\operatorname{Spec}\left(R\left[h_{1}, \ldots, h_{n+1}\right] / \operatorname{Rad}(I),\right.
$$

где $\operatorname{Rad}(I)$ - радикал идеала $I=\left(p_{n, \lambda}\right)_{\lambda \neq \varnothing}$. Таким образом, мы задали $\mathscr{M}_{n}$ идеалом полиномов в конечномерном аффинном пространстве над полем $R$, откуда следует, что $\mathscr{M}_{n}$ является многообразием при $n \neq 1$.

Теорема 1.10. Два решения $f_{1}(x)$ и $f_{2}(x)$ n-уравнения Хириебруха совпадают, если и только если $f_{1}^{(q)}(0)=f_{2}^{(q)}(0)$ для $q=2, \ldots, n+2$.

Доказательство. Как было показано выше, ряд $h(x)$ восстанавливается по набору коэффициентов $h_{1}, \ldots, h_{n+1}$. 
Лемма 1.11. Пусть $n$ нечетно, $f(x)$ является решением уравнения (1) $и$ $h_{2 i+1}=0$ для $2 i+1<n$. Тогда $f(x)$ является нечетным рядом.

Доказательство. Подставим в выражение (8) вместо функции $\tilde{h}$ ее разложение в ряд и применим оператор $L$. В результате получим разложение по базису полиномов Шура, в котором коэффициент при $s_{2 k}\left(x_{0}, \ldots, x_{n}\right)$ будет состоять из мономов, представляющих собой произведения нечетной степени с нечетным набором множителей $h_{2 k+1}$. Выбирая $h_{2 k+1}$ в качестве первого ненулевого нечетного коэффициента, мы получаем равенство нулю полинома, в котором присутствует моном $h_{2 k+1}$, а каждый из прочих мономов зависит от некоторого $h_{2 l+1}$ с меньшим индексом. Таким образом, отсюда следует равенство нулю всех последующих $h_{2 k+1}$ с $2 k+1 \geqslant n$.

В частности, для нечетности 1-жесткой функции достаточно условия $h_{1}=0$, а для случая 3 -жесткой функции достаточно, чтобы $h_{1}=h_{3}=0$, и т. д.

\section{§2. Классификация решений 2-уравнения Хирцебруха}

Теорема 2.1. Пусть ряд $f(x)$ удовлетворяет 2-уравнению Хирцебруха. Тогда

(i) ecли $C_{2} \neq 0$, mo $f(x)=\left(e^{\alpha x}-e^{\beta x}\right) /\left(\alpha e^{\beta x}-\beta e^{\alpha x}\right)$ u $C_{2}=\alpha^{2}+\alpha \beta+\beta^{2}$;

(ii) если $C_{2}=0$, mо $f(x)$ - эллиптическая функция уровня 3 или ее вырождение.

Доказательство. По теореме 1.10 любое решение уравнения (1) для $n=2$ определяется координатами $h_{1}, h_{2}, h_{3}$. Из результатов [13] следует, что для решений выполнено соотношение $h_{3}\left(h_{1}^{2}+3 h_{2}\right)=0$.

Как мы знаем, двупараметрический род Тодда задает двумерное семейство в пространстве решений $n$-уравнения Хирцебруха для любого $n$. Семейство эллиптических функций уровня 3 и их вырождений также задает двумерное семейство решений 2-уравнения Хирцебруха.

Функция $C_{2}=3\left(h_{1}^{2}+h_{2}\right)$ разделяет эти два двупараметрических семейства решений в пространстве $\mathbb{A}^{3}$, так как одно из них является специальным, а другое - нет. При этом на множестве решений выполнено соотношение $h_{3}\left(h_{1}^{2}+3 h_{2}\right)=0$, являющееся произведением двух неприводимых полиномов.

Из единственности разложения алгебраического многообразия в объединение неприводимых компонент следует, что компоненты $\left\{h_{3}=0\right\}$ и $\left\{h_{1}^{2}+3 h_{2}=0\right\}$ совпадают с описанными выше семействами решений, и других решений нет. Также отсюда следует, что на параметры $h_{1}, h_{2}, h_{3}$ нет других соотношений.

Опишем это подробнее. Пусть $h_{3}=0$. Тогда $h(x)$ имеет вид $1 / x+h_{1}+h_{2} x+$ $O\left(x^{3}\right)$. Этот ряд совпадает по модулю $x^{3}$ с разложением в ряд функции $h(x)=$ $\left(\alpha e^{\beta x}-\beta e^{\alpha x}\right) /\left(e^{\alpha x}-e^{\beta x}\right)$, если положить $h_{1}=-(\alpha+\beta) / 2, h_{2}=\left(\alpha^{2}-\beta^{2}\right) / 12$. По теореме 1.10 ряд $h(x)$ является двупараметрическим родом Тодда.

Аналогичным образом можно убедиться, что случай $h_{1}^{2}+3 h_{2}=0$ соответствует эллиптической функции уровня 3 .

\section{§3. Классификация решений 3-уравнения Хирцебруха}

Теорема 3.1. Пусть ряд $f(x)$ является решением уравнения (1) для $n=3$. Многообразие $\mathscr{M}_{3}$ состоит из трех неприводимых компонент. Ряд $h(x)=$ 
$1 / f(x)$ задает точку одной из этих трех компонент, если выполняется одно из следующих трех условий:

(i) $h_{1}=h_{3}=0$, m.e. $h(x)$ - нечетный ряд;

(ii) $h_{3}=0, h_{2}^{2}+5 h_{4}=0$;

(iii) $h_{1}^{3}+3 h_{1} h_{2}+h_{3}=0,3 h_{1}^{2} h_{2}+7 h_{2}^{2}-h_{1} h_{3}-10 h_{4}=0$.

Доказательство. По теореме 1.10 все коэффициенты $h_{k}$ являются полиномами от $h_{1}, h_{2}, h_{3}, h_{4}$. Рассмотрим разложение функции $\mathscr{S}_{n, f}$ в ряд по переменным $x_{0}, \ldots, x_{n}$ и перейдем к базису из симметрических функций от этих переменных. Коэффициенты в этом базисе будут полиномами от $h_{i}$. При степенях 7 и 9 получим одно и два нетривиальных соотношения соответственно:

$$
\begin{gathered}
-2 h_{3} h_{1}^{4}-h_{2}^{2} h_{1}^{3}-5 h_{4} h_{1}^{3}+3 h_{2}^{3} h_{1}-3 h_{3}^{2} h_{1}+15 h_{2} h_{4} h_{1}+15 h_{2}^{2} h_{3}-15 h_{3} h_{4}, \\
268 h_{3} h_{1}^{6}+134 h_{2}^{2} h_{1}^{5}+670 h_{4} h_{1}^{5}-80 h_{2} h_{3} h_{1}^{4}-442 h_{2}^{3} h_{1}^{3}+3165 h_{3}^{2} h_{1}^{3} \\
-2210 h_{2} h_{4} h_{1}^{3}-2442 h_{2}^{2} h_{3} h_{1}^{2}-150 h_{3} h_{4} h_{1}^{2}-456 h_{2}^{4} h_{1}+8169 h_{2} h_{3}^{2} h_{1} \\
-14400 h_{4}^{2} h_{1}-5160 h_{2}^{2} h_{4} h_{1}+2835 h_{3}^{3}-264 h_{2}^{3} h_{3}-4920 h_{2} h_{3} h_{4}, \\
452 h_{3} h_{1}^{6}+226 h_{2}^{2} h_{1}^{5}+1130 h_{4} h_{1}^{5}+548 h_{2} h_{3} h_{1}^{4}-404 h_{2}^{3} h_{1}^{3}+1965 h_{3}^{2} h_{1}^{3} \\
-2020 h_{2} h_{4} h_{1}^{3}-3444 h_{2}^{2} h_{3} h_{1}^{2}+3120 h_{3} h_{4} h_{1}^{2}-894 h_{2}^{4} h_{1}+4683 h_{2} h_{3}^{2} h_{1} \\
-1800 h_{4}^{2} h_{1}-4830 h_{2}^{2} h_{4} h_{1}+1296 h_{3}^{3}-4218 h_{2}^{3} h_{3}+3570 h_{2} h_{3} h_{4} .
\end{gathered}
$$

Легко проверить, что радикал идеала, порожденного этими полиномами, имеет в $\mathbb{Q}\left[h_{1}, \ldots, h_{4}\right]$ примарное разложение в сумму идеалов

$$
\left(h_{1}, h_{3}\right), \quad\left(h_{3}, h_{2}^{2}+5 h_{4}\right), \quad\left(h_{1}^{3}+3 h_{1} h_{2}+h_{3}, 3 h_{1}^{2} h_{2}+7 h_{2}^{2}-h_{1} h_{3}-10 h_{4}\right) .
$$

Первый случай по лемме 1.11 соответствует нечетным функциям.

Теорема 3.2. Любая 3-эесткая функиия совпадает с одной из следующих:

(i) функиия (2), задающая двупараметрический род Тодда;

(ii) эллиптический синус Якоби;

(iii) эллиптическая функиия уровня 4.

Доказательство. Как отмечено выше, двупараметрический род Тодда является $n$-жестким для любого $n$, в частности, для $n=3$. Тот факт, что эллиптический синус Якоби и эллиптическая функция уровня 4 являются 3-жесткими, следует из классических результатов теории эллиптических функций (см. приложение А). Проверим, что нет других 3-жестких функций.

По теореме 1.10 любое решение однозначно определяется коэффициентами $h_{i}$ с $i=1,2,3,4$. По теореме 3.1 решения лежат в аффинном пространстве с координатами $h_{1}, \ldots, h_{4}$ в объединении трех двумерных неприводимых компонент. Поскольку решения, принадлежащие этим компонентам, задаются различными формальными рядами, им соответствуют различные наборы коэффициентов $h_{1}, h_{2}, h_{3}, h_{4}$. Таким образом, из единственности разложения алгебраического многообразия в объединение неприводимых компонент следует, что других решений нет. 


\section{§4. Топологическая интерпретация решений функционального уравнения Хирцебруха}

Опишем связь задачи о многообразии решений $n$-уравнения Хирцебруха с задачей описания $n$-жестких родов Хирцебруха (см. определение 4.3). Мы дадим все необходимые понятия и сформулируем результаты. Детали см. в [14].

Рассмотрим формальный ряд $f(x)=x+\sum_{k=0}^{\infty} f_{k} x^{k+1}$ с коэффициентами $f_{k}$ из некоторого коммутативного ассоциативного кольца $R$. Тогда для любого $m$ произведение $\prod_{i=1}^{m} x_{i} / f\left(x_{i}\right)$ является симметрической функцией по переменным $x_{i}, i=1, \ldots, m$. Соответственно оно может быть выражено через элементарные симметрические функции:

$$
L_{f, m}:=L_{f, m}\left(e_{1}, \ldots, e_{m}\right)=\prod_{i=1}^{m} \frac{x_{i}}{f\left(x_{i}\right)} .
$$

Подстановка $x_{m+1} \mapsto 0$ переводит $e_{i}$ в $e_{i}$ при $i \leqslant m$ и в нуль при $i=m+1$. Таким образом, выполнено равенство $\left.L_{f, m+1}\right|_{e_{m+1}=0}=L_{f, m}$ рядов от переменных $e_{1}, \ldots, e_{m}$, хотя слева переменные $e_{i}$ зависят от $x_{1}, \ldots, x_{n}$, а справа от $x_{1}, \ldots, x_{n+1}$.

В кольце $R\left[\left[e_{1}, e_{2}, \ldots\right]\right]$ с градуировкой $\operatorname{deg} e_{i}=2 i$ элементы $L_{f, m}$ и $L_{f, m+1}$ совпадают в степенях, не превосходящих $2 m$. Таким образом, мы можем говорить о сходимости последовательности $L_{f, m}$ при $m \rightarrow \infty$. Обозначим предел через $L_{f}$.

Пусть задано $m$-мерное комплексное векторное расслоение $\xi$ над конечномерным пространством $B$. Результат подстановки в $L_{f}$ классов Чженя $c_{i}(\xi)$ вместо переменных $e_{i}$ называется комплексным характеристическим классом Хирцебруха расслоения $\xi$ (или, короче, классом Хирщебруха расслоения $\xi$ ):

$$
L_{f}(\xi)=L_{f}\left(c_{1}(\xi), \ldots, c_{m}(\xi)\right) \in \mathrm{H}^{*}(B ; R) .
$$

Так как $c_{i}(\xi)=0$ при $i>m$, то $L_{f}(\xi)=L_{f, m}(\xi)$. Комплексным характеристическим классом Хириебруха комплексного многообразия называется класс Хирцебруха его касательного расслоения.

Обратим внимание, что $c_{i}(\xi)=c_{i}(\xi \oplus \ell)$, где $\ell$ есть $l$-мерное тривиальное комплексное расслоение, $l \geqslant 1$.

Говорят, что гладкое многообразие $M^{2 n}$ имеет стабильно комплексную cтруктуру, если для некоторого $m \geqslant n$ задано комплексное $m$-мерное расслоение $\xi \rightarrow M^{2 n}$ и фиксирован изоморфизм вещественных векторных расслоений

$$
T\left(M^{2 n}\right) \oplus \mathbb{R}^{2 m-2 n} \rightarrow \xi .
$$

Комплексный род Хирцебруха $L_{f}\left(M^{2 n}\right)$ стабильно комплексного многообразия $M^{2 n}$ определен формулой

$$
L_{f}\left(M^{2 n}\right)=\left(L_{f, m}(\xi),\left\langle M^{2 n}\right\rangle\right) \in R,
$$

где $\left\langle M^{2 n}\right\rangle \in \mathrm{H}_{2 n}\left(M^{2 n}, \mathbb{Z}\right)$ - фундаментальный цикл многообразия. Соответствие $\left[M^{2 n}\right] \rightarrow L_{f}\left(M^{2 n}\right)$ задает кольцевой гомоморфизм $L_{f}: \Omega_{U} \rightarrow R$, где $\Omega_{U}-$ кольцо кобордизмов стабильно комплексных многообразий.

Обозначим через $\left(M^{2 n} ; \mathrm{T}^{k}\right)$ пару, где $M^{2 n}$ - гладкое многообразие со стабильно комплексной структурой и согласованным с этой структурой гладким действием компактного тора $\mathrm{T}^{k}$. 
Умножение на комплексные числа задает действие окружности $S^{1}=\{z \in \mathbb{C}$, $|z|=1\}$ на комплексном линейном пространстве $\mathbb{C}^{N+1}$ и, следовательно, свободное действие на сфере $S^{2 N+1}=\left\{\left(z_{1}, \ldots, z_{N+1}\right) \in \mathbb{C}^{N+1}: \sum_{k=1}^{N+1}\left|z_{k}\right|^{2}=1\right\}$. Получаем расслоение $S^{2 N+1} \rightarrow \mathbb{C} P^{N}$ со слоем $S^{1}$ и для каждого $k$ прямое произведение расслоений

$$
E_{k, N}=\prod S^{2 N+1} \rightarrow B_{k, N}=\prod \mathbb{C} P^{N}
$$

Мы можем взять индуцированные расслоения гладких многообразий $\pi_{N}: E_{k, N} \times_{\mathrm{T}^{k}} M^{2 n} \rightarrow B_{k, N}$ со слоем $M^{2 n}$.

В теории комплексных кобордизмов $U^{*}(\cdot)$ определен гомоморфизм Гизина $\pi_{N, !}: U^{*}\left(E_{k, N} \times_{T^{k}} M^{2 n}\right) \rightarrow U^{*}\left(B_{k, N}\right)$, который задает предельный гомоморфизм $\pi_{!}: U^{*}\left(E_{k} \times_{\mathrm{T}^{k}} M^{2 n}\right) \rightarrow U^{*}\left(B_{k}\right)$, где $B_{k}=\prod \mathbb{C} P^{\infty}$ и $U^{*}\left(B_{k}\right)=\Omega_{U}\left[\left[x_{1}, \ldots, x_{k}\right]\right]$.

Определение 4.1 (см. [14]). Универсальным торическим родом пары $\left(M^{2 n}, \mathrm{~T}^{k}\right)$ называется формальный ряд

$$
\Phi\left(M^{2 n} ; \mathrm{T}^{k}\right)=\pi_{!}(1) \in U^{-2 n}\left(B_{k}\right) \subset \Omega_{U}\left[\left[x_{1}, \ldots, x_{k}\right]\right] .
$$

Теорема 4.2 [14]. Имеет место формула

$$
\Phi\left(M^{2 n} ; \mathrm{T}^{k}\right)=\left[M^{2 n}\right]+\sum_{|\omega|>0} g_{\omega}\left(M^{2 n} ; \mathrm{T}^{k}\right) x^{\omega},
$$

где $x^{\omega}=x_{1}^{i_{1}} \cdots x_{k}^{i_{k}},|\omega|=i_{1}+\cdots+i_{k}$ u $g_{\omega}\left(M^{2 n} ; \mathrm{T}^{k}\right) \in \Omega_{U}^{-2(n+|\omega|)}-$ клacc комплексных кобордизмов пространства $G_{\omega}\left(M^{2 n}\right)$ расслоения $G_{\omega}\left(M^{2 n}\right) \rightarrow B_{\omega}$ со слоем $M^{2 n}$, причем $\left[B_{\omega}\right]=0 \in \Omega_{U}$.

Таким образом, для каждого рода Хирцебруха $L_{f}: \Omega_{U} \rightarrow R$ определен так называемый эквивариантный род $L_{f, \mathrm{~T}}$, сопоставляющий паре $\left(M^{2 n} ; \mathrm{T}^{k}\right)$ ряд

$$
\Phi_{f}\left(M^{2 n} ; \mathrm{T}^{k}\right)=L_{f}\left[M^{2 n}\right]+\sum_{|\omega|>0} L_{f}\left[G_{\omega}\left(M^{2 n}\right)\right] x^{\omega} .
$$

Определение 4.3. Род Хирцебруха $L_{f}$ называется $M^{2 n}$-эсестким, если

$$
\Phi_{f}\left(M^{2 n} ; \mathrm{T}^{k}\right)=L_{f}\left[M^{2 n}\right] \in R
$$

$\mathbb{C} P^{n}$-жесткий род Хирцебруха мы будем называть $n$-жестким.

Из теоремы 4.2 вытекает

Следствие 4.4. Род Хириебруха $L_{f}$ является $M^{2 n}$-жестким тогда и только тогда, когда $L_{f}\left[G_{\omega}\left(M^{2 n}\right)\right]=0$ для всех $\omega,|\omega|>0$.

Рассмотрим $(n+1)$-мерное комплексное векторное расслоение $\xi \rightarrow X$ над гладким стабильно комплексным многообразием $X$. Пространство комплексной проективизации $\mathbb{C} P(\xi) \rightarrow X$ с комплексным проективным пространством $\mathbb{C} P^{n}$ в качестве слоя имеет каноническую структуру стабильно комплексного многообразия.

Определение 4.5. Род Хирцебруха $L_{f}: \Omega_{U} \rightarrow R$ называется $n$-мультипликативным, если $L_{f}[\mathbb{C} P(\xi)]=L_{f}\left[\mathbb{C} P^{n}\right] L_{f}[X]$ для любых $(n+1)$-мерных комплексных векторных расслоений $\xi \rightarrow X$.

Теорема 4.6. Любой $n$-мулътипликативный род Хирцебруха является $\mathbb{C} P^{n}$-юсестким. 
Доказательство вытекает из того факта, что многообразия $G_{\omega}\left(\mathbb{C} P^{n}\right)$, описанные в [14], являются комплексными проективизациями расслоений над стабильно комплексными многообразиями $B_{\omega}$, такими, что $\left[B_{\omega}\right]=0$.

В случае, когда действие тора $\mathrm{T}^{k}$ на многообразии $M^{2 n}$ имеет только изолированные неподвижные точки, имеет место теорема локализации универсального торического рода $\Phi\left(M^{2 n} ; \mathrm{T}^{k}\right)$ (см. [14]) и, следовательно, теорема локализации рода $\Phi_{f}\left(M^{2 n} ; \mathrm{T}^{k}\right)$ для любого рода Хирцебруха $L_{f}$.

Каноническое действие тора $\mathrm{T}^{n+1}$ на $\mathbb{C} P^{n}$ дает пару $\left(\mathbb{C} P^{n} ; \mathrm{T}^{n+1}\right)$, для которой имеет место формула локализации

$$
\Phi_{f}\left(\mathbb{C} P^{n}\right)=\sum_{j=0}^{n} \prod_{i \neq j} \frac{1}{f\left(x_{j}-x_{i}\right)} .
$$

Следствие 4.7. Многообразие $M_{n}=\{f(x)\}$ решений $n$-уравнения Хирцебруха с $C_{n}(f)=L_{f}\left[\mathbb{C} P^{n}\right]$ совпадает с многообразием $п$-мультипликативных родов Хирчебруха $\left\{L_{f}\right\}$.

\section{Приложение А. Эллиптические функции уровня $d$}

Возьмем $\tau$ в верхней полуплоскости комплексного переменного и положим $L=2 \pi i(\mathbb{Z} \oplus \mathbb{Z} \tau)$. Существует $L$-периодическая функция $g(x)$, имеющая в начале координат нуль порядка $d$, а в некоторой точке $P$ - полюс порядка $d$ и не имеющая других нулей и полюсов по модулю $L$. В [8, приложение I, §6] доказывается существование и единственность такой функции в терминах $\theta$-функций. Известно, что тогда $P=2 \pi i(k+l \tau) / d, k, l \in \mathbb{Z}, P \neq 0$. Функция $g(x)$ при условии нормировки $g(x)=x^{d}+\cdots$ однозначно определяется точкой $P$.

При данных предположениях можно извлечь единственный корень степени $d$ с условием нормировки

$$
f(x)=\sqrt[d]{g(x)}=x+\cdots .
$$

Функция $f(x)$ является периодической относительно решетки $d L$. Такие функции называются эллиптическими уровня $d$. Заметим, что функция однозначно определена выбором решетки $L$ и точки $P$.

Опишем явно реализацию эллиптической функции уровня $d$, использованную в работе [7]. Функция

$$
\Phi(x)=\frac{\sigma(\alpha-x)}{\sigma(\alpha) \sigma(x)} e^{x \zeta(\alpha)}
$$

называется функцией Бейкера-Ахиезера, где $\sigma(x)$ - целая функция, $\sigma$-функция Вейерштрасса. Функция $\Phi(x)$ зависит от параметра $\alpha$ и параметров эллиптической кривой $g_{2}$ и $g_{3}$, имеет простой полюс при $x=0$ и простой нуль при $x=\alpha$. Рассмотрим функцию

$$
f(x)=\frac{e^{\lambda x}}{\Phi(x)} .
$$

Пусть $2 \omega_{1}, 2 \omega_{2}-$ периоды эллиптической кривой. Возьмем $\alpha=2 \frac{n}{d} \omega_{1}+2 \frac{m}{d} \omega_{2}$, $\lambda=-2 \frac{n}{d} \zeta\left(\omega_{1}\right)-2 \frac{m}{d} \zeta\left(\omega_{2}\right)+\zeta(\alpha)$, где $m, n=0, \ldots, d-1$. Тогда

$$
f\left(x+2 \omega_{1}\right)=e^{-2 \pi i n / d} f(x), \quad f\left(x+2 \omega_{2}\right)=e^{-2 \pi i m / d} f(x) .
$$


Из этих явных формул следует, что функция $f(x)$ является эллиптической уровня $d$.

Приведем разложение в ряд функции $f(x)$ до $x^{5}$ включительно:

$$
\begin{aligned}
f(x)=x+2 \lambda \frac{x^{2}}{2 !}+ & 3\left(\lambda^{2}+\wp(\alpha)\right) \frac{x^{3}}{3 !}+4\left(\lambda^{3}-\wp^{\prime}(\alpha)+3 \lambda \wp(\alpha)\right) \frac{x^{4}}{4 !}+ \\
+ & \left(5 \lambda^{4}+30 \lambda^{2} \wp(\alpha)+45 \wp^{2}(\alpha)-20 \lambda \wp^{\prime}(\alpha)-3 g_{2}\right) \frac{x^{5}}{5 !}+\ldots
\end{aligned}
$$

Определение А.1. Формальный ряд $f(x)$ над кольцом $R \otimes \mathbb{Q}$ называется рядом Гурвица, если его можно записать в виде

$$
f(x)=x+\sum_{i=1}^{\infty} \xi_{i} \frac{x^{i+1}}{(i+1) !}
$$

где $\xi_{i} \in R, i \in \mathbb{N}$.

Теорема А.2 (см. [14]). Функиия $f(x)$, определенная бормулой (9), задается рядом Гурвича над кольиом $\mathbb{Z}\left[b_{1}, b_{2}, b_{3}, b_{4}\right]$, где

$$
b_{1}=\lambda, \quad b_{2}=\wp(\alpha), \quad b_{3}=\wp^{\prime}(\alpha), \quad b_{4}=g_{2} / 2 .
$$

Напомним классическую реализацию эллиптической функции уровня 2 в терминах ю-функции Вейерштрасса. Возьмем в качестве точки $P$ один из полупериодов $\omega_{1}, \omega_{2}, \omega_{3}=\omega_{1}+\omega_{2}$. Выбирая точку $P_{k}=\omega_{k}$, получаем $g(x)=\wp(x)-$ $e_{k}$, где $e_{k}=\wp\left(\omega_{k}\right)$. Напомним, что $\left(\wp^{\prime}(z)\right)^{2}=\left(\wp(z)-e_{1}\right)\left(\wp(z)-e_{2}\right)\left(\wp(z)-e_{3}\right)$. Положим

$$
f_{k}(x)=\frac{1}{\sqrt{\wp(x)-e_{k}}}, \quad k=1,2,3 .
$$

Имеем

$$
f_{k}^{\prime}(x)=-\frac{1}{2} \frac{\wp^{\prime}(x)}{\left(\wp(x)-e_{k}\right)^{3 / 2}} .
$$

Следовательно,

$$
\left(f_{k}^{\prime}(x)\right)^{2}=\frac{\wp(x)-e_{i}}{\wp(x)-e_{k}} \cdot \frac{\wp(x)-e_{j}}{\wp(x)-e_{k}}=\left(1+\left(e_{i}-e_{k}\right) f_{k}^{2}(x)\right)\left(1+\left(e_{j}-e_{k}\right) f_{k}^{2}(x)\right),
$$

где $\{i, j, k\}=\{1,2,3\}$. Различным точкам $P_{k}$ соответствуют различные аналитические функции. Но при этом для каждого $k$ мы получаем двупараметрическое семейство функций, удовлетворяющих дифференциальному уравнению одного и того же вида $\left(f^{\prime}\right)^{2}=1+2 \delta f^{2}+\varepsilon f^{4}$, задающему эллиптический синус Якоби с параметрами $\delta$ и $\varepsilon$. Мы видим, что общее аналитическое решение этого уравнения задает единственное разложение в ряд при $x=0$, коэффициенты которого - полиномы от $\delta$ и $\varepsilon$. При этом замена точки $P$ соответствует перестановке полупериодов $\omega_{1}, \omega_{2}, \omega_{3}$. А именно, $f_{k}(x)=\operatorname{sn}\left(x ; \varepsilon_{k}, \delta_{k}\right)$, где $2 \delta_{k}=e_{i}+e_{j}-2 e_{k}, \varepsilon_{k}=\left(e_{i}-e_{k}\right)\left(e_{j}-e_{k}\right)$.

Ниже (см. лемму В.1) мы увидим, что для произвольного $d$ мы получаем одно двупараметрическое семейство функций, задающих эллиптический род уровня $d$.

Предложение А.3. Эллиптическая функиия уровня $d$ является $n$-жесткой, если d делит $n+1$. 
Доказательство. Пусть $f(x)$ - эллиптическая функция уровня $d$. Тогда для $x_{0}, \ldots, x_{n}$, различных по модулю $L$, функция

$$
F(x):=\prod_{i=0}^{n} \frac{1}{f\left(x-x_{i}\right)}
$$

для $n+1=r d$ является эллиптической. Действительно, рассмотрим сдвиги аргумента функции $F(x)$ на периоды $2 \omega_{1}, 2 \omega_{2}$. Функция $f^{d}(z+a)$ является эллиптической, поэтому при сдвиге аргумента на период функция $f(x+a)$ умножается на корень из единицы степени $d$ для любого $a$. Этот корень из единицы зависит от выбора периода, но не зависит от $a \in \mathbb{C}$. Отсюда следует, что функция $F(x)$ не меняется при сдвигах аргумента на периоды.

Поскольку функция $F(x)$ является эллиптической на кривой $\mathbb{C} / L$, сумма вычетов в ее полюсах равна нулю:

$$
0=\sum_{x \in \mathbb{C} / \Lambda} \operatorname{res}_{x} F=\frac{1}{f\left(x_{0}-x_{1}\right) \cdots f\left(x_{0}-x_{n}\right)}+\cdots+\frac{1}{f\left(x_{n}-x_{0}\right) \cdots f\left(x_{n}-x_{n-1}\right)} .
$$

Заметим, что это равенство выполнено в кольце формальных рядов от переменных $x_{i}$, поэтому оно выполнено для любых $x_{i}$, а не только при различных по модулю $L$. Последнее условие равносильно $n$-жесткости.

\section{Приложение В. Преобразования эллиптических функций и модулярные формы}

Рассмотрим пару функций

$$
\operatorname{sn}^{2}(x, k)=\varphi(x), \quad \operatorname{sn}^{2}(x / M, \lambda)=\psi(x) .
$$

Когда эти функции алгебраически зависимы? Пусть функции $\varphi(x)$ и $\psi(x)$ имеют соответственно периоды $2 \omega_{1}, 2 \omega_{2}$ и $2 \tilde{\omega}_{1}, 2 \tilde{\omega}_{2}$. Положим $L_{1}=\left\langle 2 \omega_{1}, 2 \omega_{2}\right\rangle$, $L_{2}=\left\langle 2 \tilde{\omega}_{1}, 2 \tilde{\omega}_{2}\right\rangle$. Из алгебраической зависимости функций $\varphi(x)$ и $\psi(x)$ следует (см. [11, гл. VI]), что $L_{1} \otimes \mathbb{Q}=L_{2} \otimes \mathbb{Q}$. Более того, эти условия оказываются равносильными.

Можно ограничиться рассмотрением только преобразований вида

$$
\omega_{1}=\alpha \tilde{\omega}_{1}+\beta \tilde{\omega}_{2}, \quad \omega_{2}=\gamma \tilde{\omega}_{1}+\delta \tilde{\omega}_{2}
$$

с целыми $\alpha, \beta, \gamma, \delta$. Тогда площадь параллелограмма периодов, натянутого на $\omega_{1}, \omega_{2}$, в $m=\left|\begin{array}{ll}\alpha & \beta \\ \gamma & \delta\end{array}\right|$ раз больше площади параллелограмма периодов, натянутого на $\tilde{\omega}_{1}, \tilde{\omega}_{2}$. Такое преобразование называется преобразованием степени $\mathrm{m}$. Известно, что преобразование степени 1 является композицией преобразований

$$
\begin{array}{ll}
\omega_{1}=\tilde{\omega}_{1}+\tilde{\omega}_{2}, & \omega_{2}=\tilde{\omega}_{2}, \\
\omega_{1}=-\tilde{\omega}_{1}, & \omega_{2}=\tilde{\omega}_{2} .
\end{array}
$$

Оказывается, что любое преобразование степени $m$ является композицией преобразований степени 1 и следующих преобразований (называемых главными преобразованиями степени $m$ ):

$$
\left\{\begin{array} { l } 
{ \omega _ { 1 } = m \tilde { \omega } _ { 1 } , } \\
{ \omega _ { 2 } = \tilde { \omega } _ { 2 } }
\end{array} \text { или } \quad \left\{\begin{array}{l}
\omega_{1}=\tilde{\omega}_{1} / m, \\
\omega_{2}=\tilde{\omega}_{2}
\end{array}\right.\right.
$$


И

$$
\left\{\begin{array} { l } 
{ \omega _ { 1 } = \tilde { \omega } _ { 1 } , } \\
{ \omega _ { 2 } = m \tilde { \omega } _ { 2 } }
\end{array} \quad \text { или } \quad \left\{\begin{array}{l}
\omega_{1}=\tilde{\omega}_{1}, \\
\omega_{2}=\tilde{\omega}_{2} / m,
\end{array}\right.\right.
$$

где $m$ - натуральное число. В первом случае преобразование состоит в изменении первого периода, а во втором - в изменении второго.

Как мы видели выше, в случае эллиптической функции уровня 2 мы получаем функции $f_{k}$, удовлетворяющие дифференциальному уравнению $\left(f_{k}^{\prime}\right)^{2}=$ $1+2 \varepsilon f_{k}^{2}+\delta f_{k}^{4}$. Параметры $\varepsilon, \delta$ выражаются через $e_{1}, e_{2}, e_{3}$. Преобразование степени 1 переставляет $e_{1}, e_{2}, e_{3}$. Таким образом, группа преобразований степени 1 действует на пространстве параметров дифференциального уравнения, переставляя частные аналитические решения уравнения. Это действие нетривиально, однако, несмотря на свободу выбора одной из трех точек $P$, мы получаем одно универсальное решение дифференциального уравнения, где параметры $\varepsilon$, $\delta$ являются независимыми переменными.

Чтобы распространить рассуждение на случай эллиптических функций уровня $d$, напомним сначала некоторые понятия.

Рассмотрим решетку $L=\langle 1, \tau\rangle$, где $\operatorname{Im}(\tau)\rangle 0$. На верхней полуплоскости группа $\mathrm{SL}(2, \mathbb{R})$ действует дробно-линейными преобразованиями. Подгруппа $\mathrm{SL}(2, \mathbb{Z})$, сохраняющая $L$, называется модулярной группой. Модулярной формой веса $k$ для модулярной группы Г называется функция $f$ на верхней полуплоскости, для которой

$$
f(g x)=(c \tau+d)^{k} f(x)
$$

для любых $g=\left(\begin{array}{ll}a & b \\ c & d\end{array}\right) \in \Gamma$, голоморфная во всех параболических точках. Рассматриваются не только группы автоморфизмов заданной решетки $L$, но и некоторые другие подгруппы в $\mathrm{SL}(2, \mathbb{Z})$ конечного индекса. Модулярные формы нечетного веса равны нулю. Модулярные формы четного веса задаются рядами

$$
G_{2 k}=\sum_{(m, n) \neq(0,0)} \frac{1}{(m+n \tau)^{2 k}} .
$$

Модулярные инварианты определяются как

$$
g_{2}=60 G_{4}, \quad g_{3}=140 G_{6} .
$$

Известно, что любая модулярная форма выражается как полином от $g_{2}, g_{3}$.

Предложени В.1. Семейство эллиптических функиий уровня d двупараметрическое. В качестве двух независимых параметров могут быть (неканонически) выбраны $g_{2}, g_{3}$.

Доказательство. Пусть эллиптическая функция $f(x)$ уровня $d$ задается формулой $(9)$ и соответствует решетке $L=\left\langle 2 \omega_{1}, 2 \omega_{2}\right\rangle$ с базисом $\left\{2 \omega_{1}, 2 \omega_{2}\right\}$ и точке $P=2 \frac{m}{d} \omega_{1}+2 \frac{n}{d} \omega_{2}$, которую далее для краткости будем называть $(m, n)$ точкой. Мы можем заменить базис в решетке $L$ таким образом, чтобы в новом базисе точка $P$ была $(k, 0)$-точкой для некоторого целого $k$, взаимно простого с $d$. Обозначим через $\tilde{f}(x)$ функцию, определенную в новом базисе той же решетки и для той же точки $P$, которая в новом базисе является $(k, 0)$-точкой. 
Функции $f^{d}(x)$ и $\tilde{f}^{d}(x)$ являются $L$-периодическими. Нули этих функций расположены в точках решетки $L$ и имеют порядок $d$, полюсы расположены в точках $P+L$ и также имеют порядок $d$. Отсюда и из условий $f^{d}(x)=x^{d}+O\left(x^{d+1}\right)$ и $\tilde{f}^{d}(x)=x^{d}+O\left(x^{d+1}\right)$ следует, что $f^{d}(x)=\tilde{f}^{d}(x)$. Нормировка функций при $x=0$ дает равенство $f(x)=\tilde{f}(x)$.

Из формулы (10) следует, что функция $f(x)$, определенная для $(k, 0)$-точки $P$, не меняется при сдвиге аргумента на период $2 \omega_{2}$. Выберем такие целые числа $n_{1}$ и $n_{2}$, что $k n_{2}-d n_{1}=1$. При сдвиге точки $P$ на период $2 \omega_{2}$ мы получаем, что в базисе $\left\{2 k \omega_{1}+2 d \omega_{2}, 2 n_{1} \omega_{1}+2 n_{2} \omega_{2}\right\}$ точка $P$ становится $(1,0)$-точкой. Таким образом, замена базиса решетки и, возможно, сдвиг точки $P$ на период, позволяет ограничиться только $(1,0)$-точкой $P$, т. е. та же функция $f(x)$ может быть явно задана формулой (9) при той же решетке $L$ с другим базисом. Таким образом, коэффициенты ряда $f(x)$ могут быть выражены через параметры $g_{2}$, $g_{3}$ решетки $L$.

Замечание В.2. Функция $f^{d}(x)$ является эллиптической, и в ее разложении в ряд все коэффициенты являются полиномами от $g_{2}, g_{3}$. В то же время, уже коэффициент при $x^{2}$ ряда $f(x)$ может быть не полиномом от $g_{2}, g_{3}$, а функцией от них.

Пример В.3. Рассмотрим случай $d=4$. Как мы знаем, эллиптической функцией уровня 2 является только эллиптический синус Якоби $\operatorname{sn}(x)$. Начиная с решетки $L=\left\langle 2 K, 2 i K^{\prime}\right\rangle$, мы получаем, что $\operatorname{sn}(x)$ преобразуется при сдвигах аргумента следующим образом:

$$
\operatorname{sn}(x+2 K)=-\operatorname{sn}(x), \quad \operatorname{sn}\left(x+2 i K^{\prime}\right)=\operatorname{sn}(x) .
$$

Применяя деление первого периода на два, известное как преобразование Ландена (см. [11, гл. VI, §38]), мы можем получить явное выражение для функции $M \operatorname{sn}(x / M ; \lambda)$, а именно,

$$
M \operatorname{sn}\left(\frac{x}{M} ; \lambda\right)=\operatorname{sn}(x ; k) \sqrt{\frac{1-\operatorname{sn}^{2}(x ; k)}{1-k \operatorname{sn}^{2}(x ; k)}}=\frac{\operatorname{sn}(x ; k) \operatorname{cn}(x ; k)}{\operatorname{dn}(x ; k)},
$$

где $M=1 /\left(1+k^{\prime}\right)=\operatorname{sn}^{2}(K / 2 ; k), \lambda=\left(1-k^{\prime}\right) /\left(1+k^{\prime}\right)$. Из этого выражения видно, что функция $M^{2} \mathrm{sn}^{2}(x / M ; \lambda)$ является двоякопериодической относительно $L^{\prime}=$ $\left\langle K, 2 i K^{\prime}\right\rangle$ с нулями только в точках решетки $L^{\prime}$ и полюсами только в точках множества $K / 2+L^{\prime}$, все нули и полюсы степени 2 .

Рассмотрим функцию $M \operatorname{sn}(x / M ; \lambda)$ относительно решетки $L$. Она является двоякопериодической и в параллелограмме периодов имеет два простых нуля в 0 и $K$ и два простых полюса в $K / 2$ и $3 K / 2$. Тогда функция

$$
M^{2} \operatorname{sn}^{2}(x / M ; \lambda) \operatorname{sn}^{2}(x ; k) \operatorname{sn}^{2}(x-K / 2 ; k)
$$

имеет нули только в точках решетки $L$, полюсы только в точках множества $K / 2+L$, все нули и полюсы степени 4 . Обозначим через $\mathscr{L}$ преобразование Ландена (т. е. $\mathscr{L}(\operatorname{sn}(x ; k))=M \operatorname{sn}(x / M ; \lambda))$.

Пусть $f_{2}(x)=\operatorname{sn}(x ; k)$ - эллиптическая функция уровня 2 , соответствующая выбору точки $P$ с $(m, n)=(1,0)$. Тогда получаем эллиптическую функцию уровня 4:

$$
f_{4}(x)=\frac{1}{\sqrt{2}} \sqrt{\mathscr{L}\left(f_{2}(x)\right) f_{2}(x) f_{2}(x-K / 2)} .
$$


С другой стороны, функция $f_{2}(x)$ восстанавливается из $f_{4}(x)$ по формуле

$$
f_{2}(x)=f_{4}(x) f_{4}(x+K / 2),
$$

как следует из единственности функции $f_{2}(x)$ с заданными нулями и полюсами при условии нормировки. Таким образом, при помощи преобразования Ландена устанавливается взаимно однозначное соответствие между семействами эллиптических функций уровня 2 и уровня 4 .

Замечание B.4. Описанное выше соответствие между эллиптическими функциями уровня 2 и эллиптическими функциями уровня 4 зависит от выбора точек порядка 2 и 4. Явная формула этого соответствия может измениться.

\section{ЛитеРАТУРА}

[1] V. M. Buchstaber, A. P. Veselov, On a remarkable functional equation in the theory of generalized Dunkl operators and transformations of elliptic genera, Math. Z., 223:4 (1996), 595-607.

[2] V. M. Buchstaber, G. Felder, A. P. Veselov, Elliptic Dunkl operators, root systems, and functional equations, Duke Math. J., 76:3 (1994), 885-911.

[3] В. М. Бухштабер, А. П. Веселов, Операторы Данкла, функциональнье уравнения и преобразования эллиптических родов, УМН, 49:2(296) (1994), 147-148.

[4] Ф. Хирцебрух, Топологические методь в алгебраической геометрии, Мир, М., 1973.

[5] O. R. Musin, On rigid Hirzebruch genera, Mosc. Math. J., 11:1 (2011), 139-147.

[6] W. Fulton, Young Tableaux. With Applications to Representation Theory and Geometry, Cambridge Univ. Press, Cambridge, 1997.

[7] И. М. Кричевер, Обобщеннъе эллиптические родъ и функции Бейкера-Ахиезера, Матем. заметки, 47:2 (1990), 34-45.

[8] F. Hirzebruch, Th. Berger, R. Jung, Manifolds and Modular Forms, Fiedr. Vieweg \& Sons, Braunschweig, 1992.

[9] Э. Т. Уиттекер, Дж. Н. Ватсон, Курс современного анализа, ч. 2. Трансцендентные функиии, Физматлит, М., 1963.

[10] В. М. Бухштабер, Е. Ю. Бунькова, Формалънъе группљ Кричевера, Функц. анализ и его прил., 45:2 (2011), 23-44.

[11] Н. И. Ахиезер, Элементы теории эллиптических функций, Наука, М., 1970.

[12] В. М. Бухштабер, Е. Ю. Бунькова, Универсалъная формальная группа, определяющая эллиптическую функиию уровня 3, Чебышевский сб., 16:2 (2015), 66-78.

[13] В. М. Бухштабер, Е. Ю. Бунькова, Многообразия решений функциональных уравнений Хирцебруха, в кн.: Современные проблемы математики, механики и математической физики, Сб. статей, Тр. МИАН, т. 290, МАИК, М., 2015, 136-148.

[14] V. M. Buchstaber, T. E. Panov, Toric Topology, Amer. Math. Soc., Providence, RI, 2015.

[15] В. Н. Сачков, Введение в комбинаторные методы дискретной математики, МЦНМО, 2004.

[16] С. П. Новиков, Операторы Адамса и неподвижные точки, Изв. АН СССР, сер. матем., 32:6 (1968), 1245-1263.

Математический институт имени В. А. Стеклова РАН e-mail: buchstab@mi.ras.ru

Поступила в редакцию

Математический институт имени В. А. Стеклова РАН 5 октября 2015 г.

e-mail: netay@iitp.ru 